\title{
Cycle biologique de Pterygodermatites desportesi (Chabaud et Rousselot, 1956) Nematoda Rictulariidae
}

\author{
par J.-C. QUENTIN
}

[Station Expérimentale de La Maboké par M'Baiki (République Centrafricaine)

[Laboratoire de Zoologie (Vers) associé au C.N.R.S. (Pr A.-G. Снaвaud)

Muséum National d'Histoire Naturelle, 57, rue Cuvier, 75 - Paris, 5]

\section{Résumé}

Cycle biologique du Nématode Rictulaire Pterygodermatites desportesi (Chabaud et Rousselot, 1956), dont l'adulte est parasite d'un Rongeur Muridae d'Afrique Equatoriale Lophuromys sikapusi Tem. Les 3 premiers stades larvaires du parasite évoluent expérimentalement dans la paroi intestinale de Dermaptères Anisolabis annulipes Lucas et Diaperasticus erythrocephalus Olivier. Un $4^{\circ}$ stade femelle et un jeune mâle du Spiruride sont obtenus après quatre jours de développement chez l'hôte définitif.

\section{Summary}

Life cycle of the nematod Rictulariidae Pterygodermatites desportesi (Chabaud et Rousselot, 1956). The adult is a parasite of Muridae from equatorial Africa Lophuromys sikapusi. The experimental development of three first larval stages occurs in the intestinal wall of the Dermapters Anisolabis annulipes Lucas and Diaperasticus erythrocephalus Olivier. A 4th female stage and a young male of the Spirurid are obtained after 4 days of development in the definitive host. 
La femelle holotype de cette espèce a été découverte dans l'intestin d'un Lophuromys sikapusi Tem. du Jardin Zoologique de Brazzaville par Chabaud et Rousselot en 1956.

Les autopsies de Lophuromys piégés à Boukoko (R.C.A.) le 23 octobre 1963 et le 21 novembre 1964 permettaient de récolter successivement $22 \&$ plus $1 \delta^{*}$ et $1 q$ plus $1 \delta^{*}$ du parasite et de compléter la connaissance de l'espèce par la description du mâle (Quentin, 1966).

L'étude de l'helminthofaune des Rongeurs piégés en République Centrafricaine a en outre montré que $P$. desportesi ne parasite que le Lophuromys sikapusi. Ce Rictulaire diffère des autres espèces congénères récoltées chez les Rongeurs africains par trois caractères qui traduisent selon nous un faible degré d'évolution : capsule buccale à paroi mince, légèrement inclinée vers la face dorsale, éléments cuticulaires en petit nombre (44 paires chez le mâle, 76 paires chez la femelle dont 38 sont prévulvaires), disposition primitive des papilles cloacales du mâle de type Ascaridida, les paires 1-4-8 étant déjetées latéralement.

\section{Cycle biologique}

\section{Récolte du matériel.}

9 Lophuromys sur 41 capturés aux alentours de la Station de la Maboké sont parasités par $P$. desportesi. Trois Rongeurs piégés durant les mois de juillet et août 1968 hébergent des femelles suffisamment gravides pour permettre une infestation.

Les œufs embryonnés sont donnés en nourriture à des Dermaptères des espèces Anisolabis annulipes Lucas et Diaperasticus erythrocephalus Olivier * récoltés autour de la Station et élevés à la température ambiante moyenne de $22^{\circ} \mathrm{C}$.

Sur 40 Forficules disséquées, 11 sont parasitées par des stades larvaires de Rictulaires.

\section{Allure du développement chez l'Insecte.}

Les observations sont effectuées 24 h, 4 jours, 6 jours, 11 jours, 17 jours, 24 jours, 30 jours et 32 jours après l'infestation.

- La larve enfermée dans l'œuf présente une très grosse ampoule excrétrice. Cet organe est toujours visible sur les larves du premier stade qui ont pénétré dans la paroi de l'intestin postérieur de l'Insecte $24 \mathrm{~h}$ après l'infestation. Ces larves sont de petite taille $(280 \mu)$, leur croissance est lente jusqu'au $4^{\circ}$ jour où elles n'atteignent que $290 \mu$. Au $6^{e}$ jour de leur développement, elles mesurent $380 \mu$ et $420 \mu$ au $7^{\circ}$ jour.

- Le $11^{\circ}$ jour, la larve ébauche la première mue, la cuticule est décollée dans la région céphalique et caudale. Cette larve longue de $420 \mu$ poursuit sa croissance et atteint $550 \mu$ de long le $17^{\circ}$ jour.

\footnotetext{
* Nous remercions vivement M. le Professeur Chopard, du Muséum d'Histoire Naturelle, qui a bien voulu nous déterminer les Dermaptères.
} 
- La seconde mue survient entre le $17^{\circ}$ et le $24^{\circ}$ jour. Les larves du troisième stade mesurent 680 à $720 \mu$ de long.

\section{Morphologie des stades larvaires chez l'Insecte.}

Premier STADE: La larve du premier stade recueillie $24 \mathrm{~h}$ après l'infestation est très fine et transparente (fig. $1 \mathrm{~B}, \mathrm{C}$ ). La région céphalique est moins différenciée que celle d'autres premiers stades des Spirurides Protospirura, Gongylonema. L'épine cuticulaire est en effet réduite, les deux râpes cuticulaires ne sont pas visibles. Cette larve, par contre, possède deux glandes céphaliques analogues à celles observées sur les premiers stades de Subulures. La cellule excrétrice se présente comme une ampoule réfringente entourée de canalicules radiaires. L'œsophage est étroit, enflé dans ses régions médianes et postérieures. L'intestin a des parois minces.

Les dimensions de cette larve sont: longueur $273 \mu$, largeur 10-11 $\mu$, pore excréteur situé à $80 \mu$ de l'apex, pré-œsophage long de $30 \mu$, œsophage long de $92 \mu$, longueur de l'intestin $112 \mu$, cellule génitale à $200 \mu$ de l'apex, longueur de la queue $28 \mu$.

- La larve âgée de 4 jours s'est épaissie (fig. 1 D). Ses dimensions sont : longueur $290 \mu$, largeur $20 \mu$, anneau nerveux situé entre 40 et $50 \mu$ de l'apex, le canalicule de la cellule excrétrice débouche à $73 \mu$ de l'apex, pré-œsophage long de $37 \mu$, œsophage long de $76 \mu$, longueur de l'intestin $130 \mu$, ébauche génitale à $180 \mu$ de l'extrémité antérieure, longueur de la queue $50 \mu$.

— La larve de 6 jours a augmenté de taille (fig. 1 E). L'œsophage présente toujours les renflements médians et postérieurs. L'intestin se charge de réserves.

Ses dimensions sont : longueur $380 \mu$, largeur $24 \mu$, anneau nerveux et pore excréteur respectivement situés à 45 et $90 \mu$ de l'apex, pré-œsophage long de $36 \mu$, œsophage long de $103 \mu$, longueur de l'intestin $187 \mu$, ébauche génitale longue de $15 \mu$ située à $255 \mu$ de l'apex, longueur de la queue $56 \mu$.

DeUXIÈmE STADE: Une larve obtenue 11 jours après l'infestation a peu augmenté de taille par rapport à la larve âgée de 6 jours (fig. 2 A, B). Elle appartient cependant au second stade larvaire par son organisation interne. Sous l'exuvie du premier stade, la queue n'est plus arrondie mais pointue. L'œsophage devient cylindrique et perd les deux renflements médians et postérieurs. La limite entre les régions musculaire et glandulaire de l'œsophage n'est pas encore discernable.

Les dimensions de cette larve sont : longueur $420 \mu$, largeur $29 \mu$, anneau nerveux, pore excréteur respectivement situés à 75 et $105 \mu$ de l'apex, région pharyngienne courte $(6 \mu)$ et peu chitinisée, longueur totale de l'œsophage $144 \mu$, intestin long de $225 \mu$, ébauche génitale située à $650 \mu$ de l'apex.

— L'organisation interne de la larve de 17 jours révèle un très grand nombre de noyaux. L'anneau nerveux est nettement localisé par la condensation cellulaire qui l'entoure (fig. 2 D).

Les dimensions sont : longueur $550 \mu$, largeur $37 \mu$, anneau nerveux et pore excréteur situés respectivement à $68 \mu$ et $112 \mu$ de l'apex, vestibule buccal profond de $10 \mu$, 


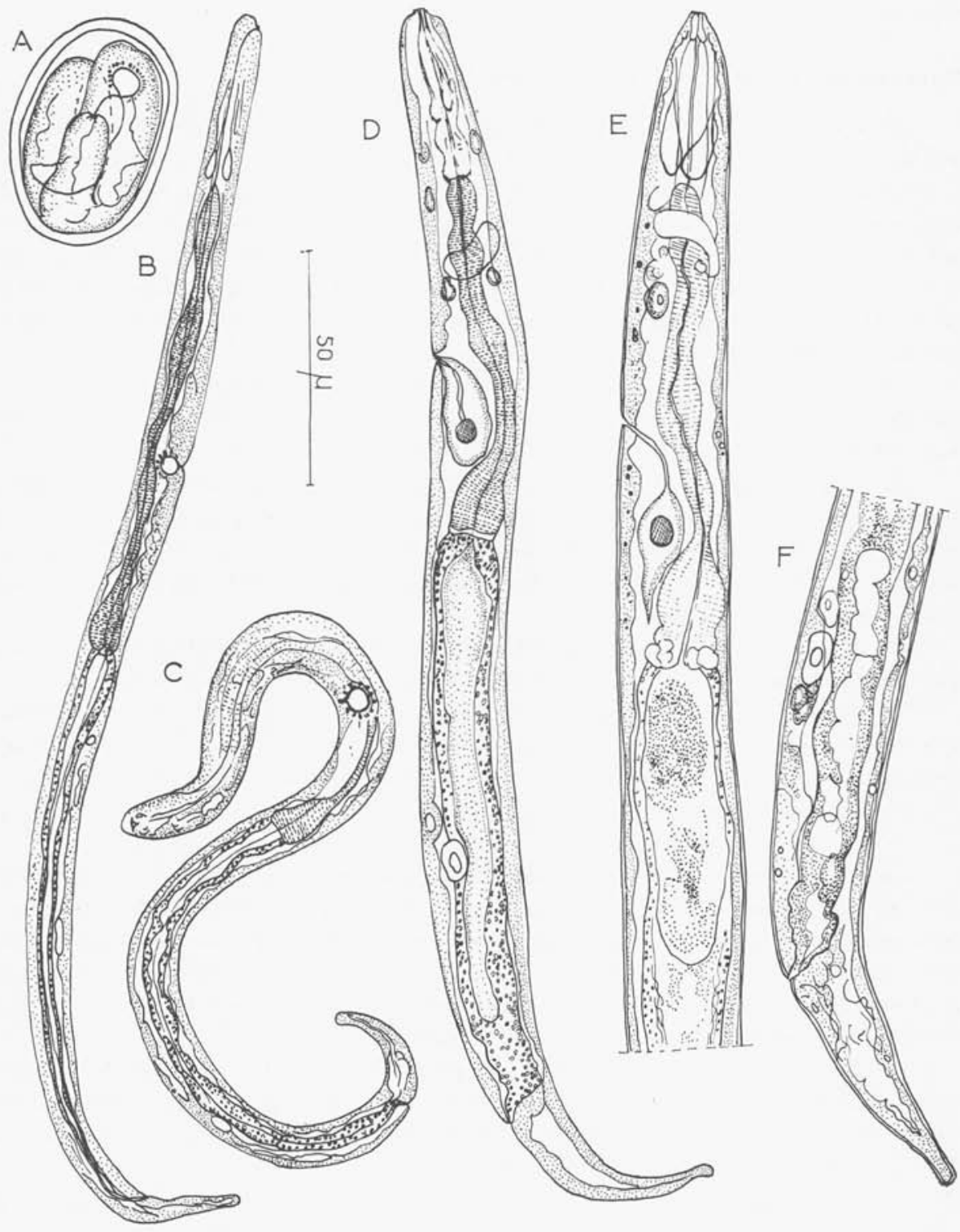

FIG. 1. - A : œuf embryonné. B, C: larves du $1^{\text {er }}$ stade 24 h après l'infestation, vues latérales. D : larve du $1^{\text {er }}$ stade 4 jours après l'infestation, vue latérale. E, F: larve du $1^{\text {er }}$ stade 6 jours après l'infestation, vue latérale. A, B, C, D, E, F : éch. $50 \mu$ 


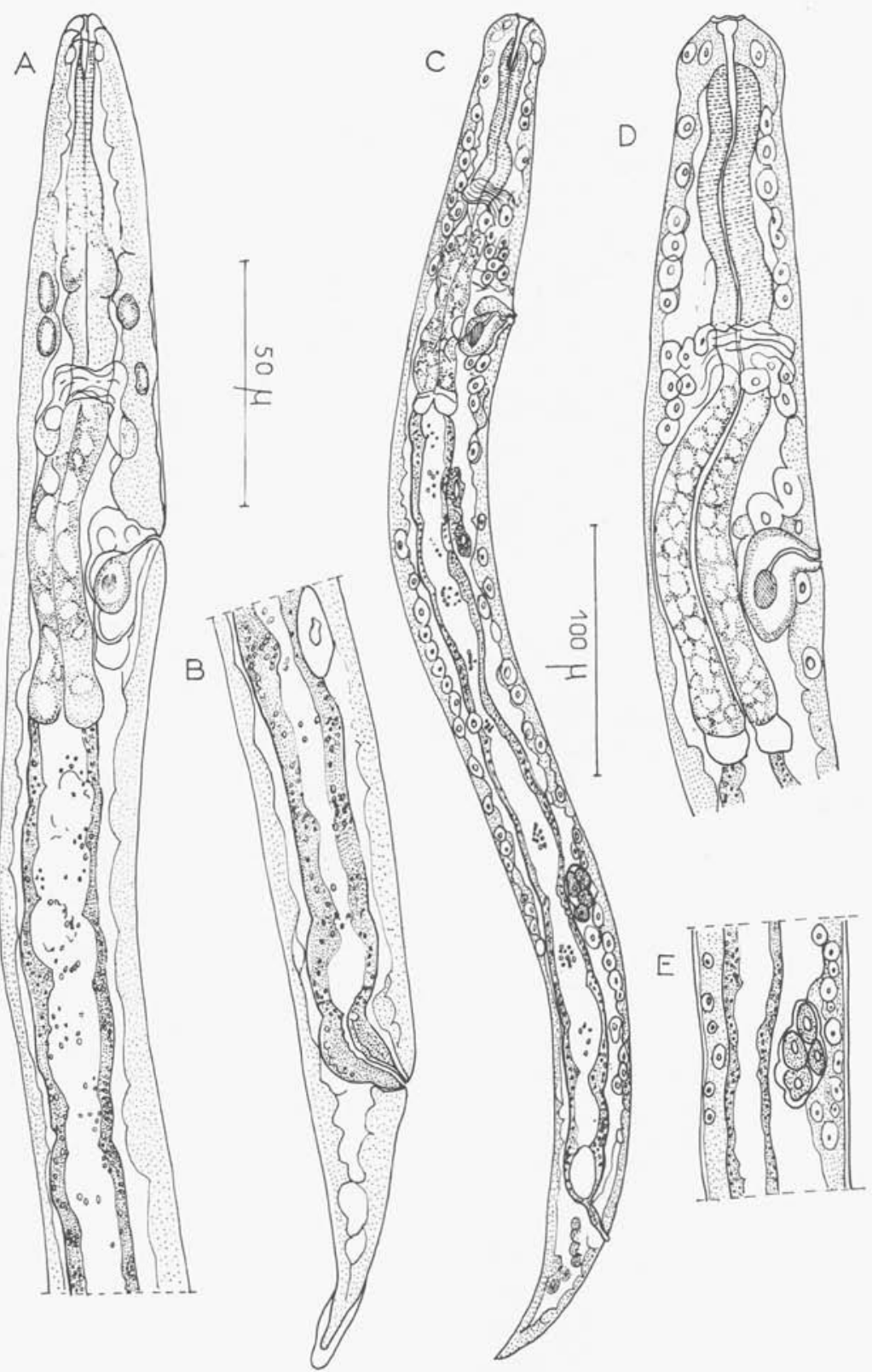

Fig. 2. - A, B: larve de 11 jours après l'infestation, décollement de l'exuvie du $1^{\text {er }}$ stade dans les régions céphalique et caudale. $\mathrm{C}$ : larve du $2^{\circ}$ stade, vue latérale. D: détail de l'extrémité antérieure de la larve du $2^{\circ}$ stade. E : détail de l'ébauche génitale. A, B, D, E : éch. $50 \mu$. C : éch. $100 \mu$ 

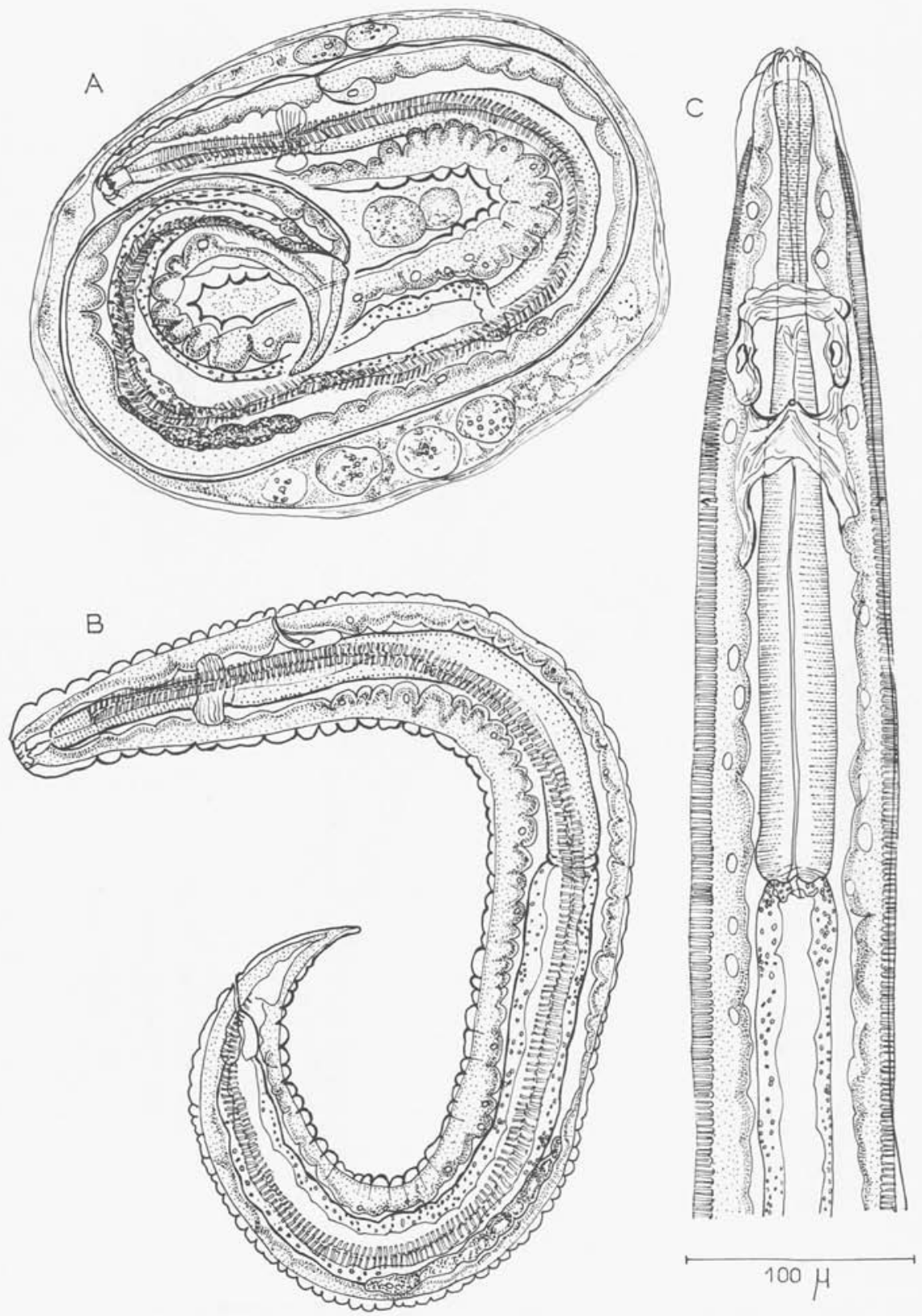

FIG. 3 - A : larve du $3^{\text {e }}$ stade encapsulée dans la paroi intestinale. B: larve dégagée de sa capsule, vue latérale. C: détail de l'extrémité antérieure de la larve du $3^{\text {e }}$ stade étalée en vue ventrale entre lame et lamelle. A, B, C : éch. $100 \mu$ 
longueur de l'œsophage musculaire $70 \mu$, de l'œsophage glandulaire $80 \mu$, longueur de l'intestin $170 \mu$, ébauche génitale multicellulaire longue de $21 \mu$ située à $340 \mu$ de l'apex (fig. $2 \mathrm{E}$ ), longueur de la queue $70 \mu$.

TroIsIÈME STADE : La larve du troisième stade est caractérisée par l'apparition de deux ailes longitudinales finement striées et d'une capsule buccale.

La structure céphalique du troisième stade présente les caractères généraux de l'adulte sans en avoir subi les déformations évolutives. Une vue apicale (fig. 4 A) montre une ouverture buccale terminale, 6 papilles du cycle interne réunies entre elles par une très fine couronne d'ornementations cuticulaires, 2 amphides, 4 papilles du cycle labial externe accolées individuellement aux 4 papilles du cycle céphalique. Sous l'ouverture buccale s'ébauche la couronne de denticules péribuccaux qui se différencient au quatrième stade. Plus en profondeur, les trois dents œsophagiennes d'égale importance sont denticulées.

Cette morphologie est différente de celle de l'adulte dont la capsule buccale est déportée dorsalement et où les terminaisons sensorielles sont saillantes avec peu d'ornementations cuticulaires. Les dents œsophagiennes de l'adulte sont représentées par une dent dorsale proéminente et lisse et deux dents latéro-ventrales de plus faible importance, très légèrement denticulées.

- La larve du troisième stade âgée de 24 jours est enfermée dans une capsule conjonctive de 200 à $280 \mu$ de diamètre (fig. 3 A) dans la paroi de l'intestin postérieur de l'Insecte. La larve y est repliée sur elle-même, fortement contractée sur le bord dorsal.

Ses dimensions sont : longueur $720 \mu$, largeur $65 \mu$ (fig. 3 B), anneau nerveux, pore excréteur respectivement situés à 90 et $130 \mu$ de l'apex, diamètre de l'ouverture buccale $12 \mu$, profondeur de la capsule $10 \mu$., longueur de l'œsophage musculaire $90 \mu$, longueur de l'œsophage glandulaire $200 \mu$ (fig. 3 C), longueur de l'intestin $350 \mu$, ébauche génitale longue de $115 \mu$ située à $420 \mu$ de l'apex, longueur de la queue $115 \mu$.

Cette larve ne subit plus de modifications. Les longueurs des différentes larves du troisième stade obtenues après 30 et 32 jours chez l'Insecte varient de $680 \mu$ à $725 \mu$.

RECHERCHE DU QUATRIÈME STADE ET DE L'ADULTE CHEZ L'HôTE DÉFINITIF : le cycle étant entièrement réalisé à la Station de La Maboké, quatre larves de troisième stade de $P$. desportesi sont données à un Praomys morio capturé sur place. Ce Muridae n'est jamais parasité spontanément par $P$. desportesi. Le Rongeur est disséqué $108 \mathrm{~h}$ après : une larve femelle du quatrième stade prête à muer et un jeune mâle sont retrouvés au niveau de l'intestin.

QuATrième STADE FEMElle (fig. $4 \mathrm{~A}$ ) : cette larve est à la fin du quatrième stade, car la cuticule est décollée dans les régions céphalique et caudale (fig. $4 \mathrm{~B}, \mathrm{C}$ ). La cuticule, ornée de deux ailes longitudinales striées, enferme encore le Nématode.

Ses dimensions sont : longueur $1.780 \mu$, largeur $130 \mu$, anneau nerveux, pore excréteur et deirides situés respectivement à 200,212 et $250 \mu$ de l'apex, longueur de l'œso- 
phage musculaire $230 \mu$, de l'œsophage glandulaire $420 \mu$, vulve située à $1.260 \mu$ de l'apex, longueur totale des deux branches utérines $850 \mu$, longueur de la queue $70 \mu$.
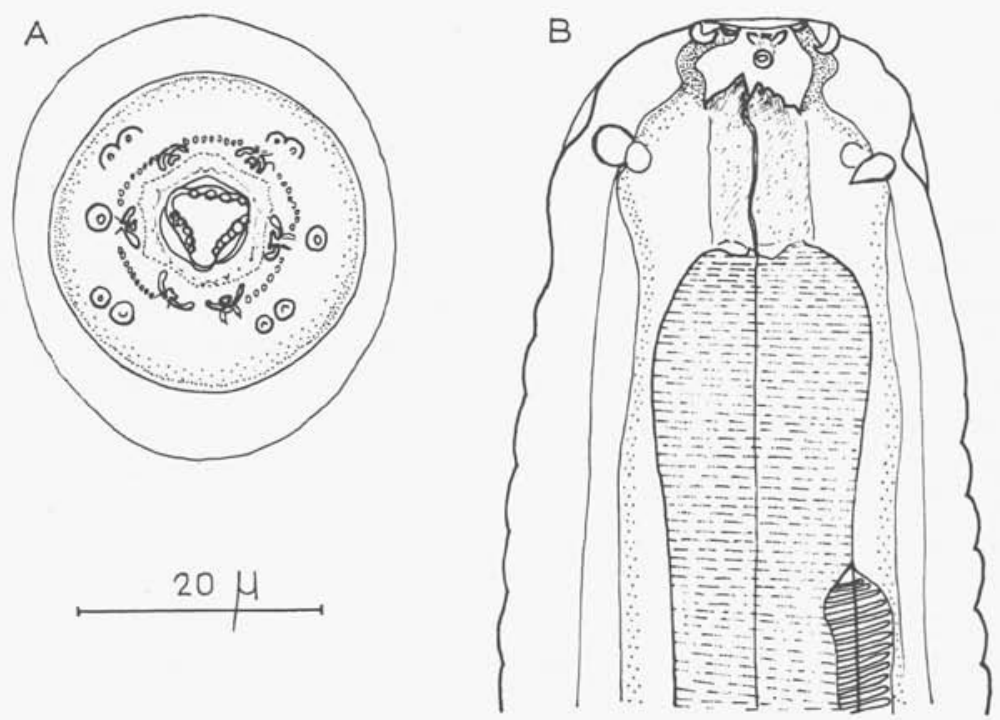

Fig. 4. - A : vue apicale d'une larve du $3^{\text {e }}$ stade chez l'Insecte, détail des terminaisons sensorielles et de l'ornementation céphalique. B : extrémité antérieure de la larve, vue latérale droite. A, B : éch. $20 \mu$

MÂle (fig. $5 \mathrm{~A}$ ): sa morphologie concorde avec celle du mâle déjà décrit de $P$. desportesi, par sa structure céphalique, et le nombre de paires d'éléments cuticulaires (44).

Ses dimensions sont : longueur $1.725 \mu$, largeur dans la région moyenne du corps $87 \mu$, anneau nerveux, pore excréteur et deirides respectivement situés à 136,200 et $210 \mu$ de l'apex, capsule céphalique profonde de $15 \mu$, œsophage musculaire long de $130 \mu$, œsophage glandulaire de $440 \mu$, spicules inégaux, le droit mesure $29 \mu$ de long, le gauche $67 \mu$.

\section{Discussion.}

Les cycles biologiques de Rictulariidae sont actuellement connus chez trois espèces :

- Rictularia cristata Froelich, $1902=R$. amurensis Schulz, 1927, parasite de Rongeurs Glirididae, Microtidae et Muridae d'Europe continentale et de Russie, évolue d'après Morosov 1960, jusqu'au troisième stade chez un Diplopode Chromatoiulus projectus kochi (Vher). 


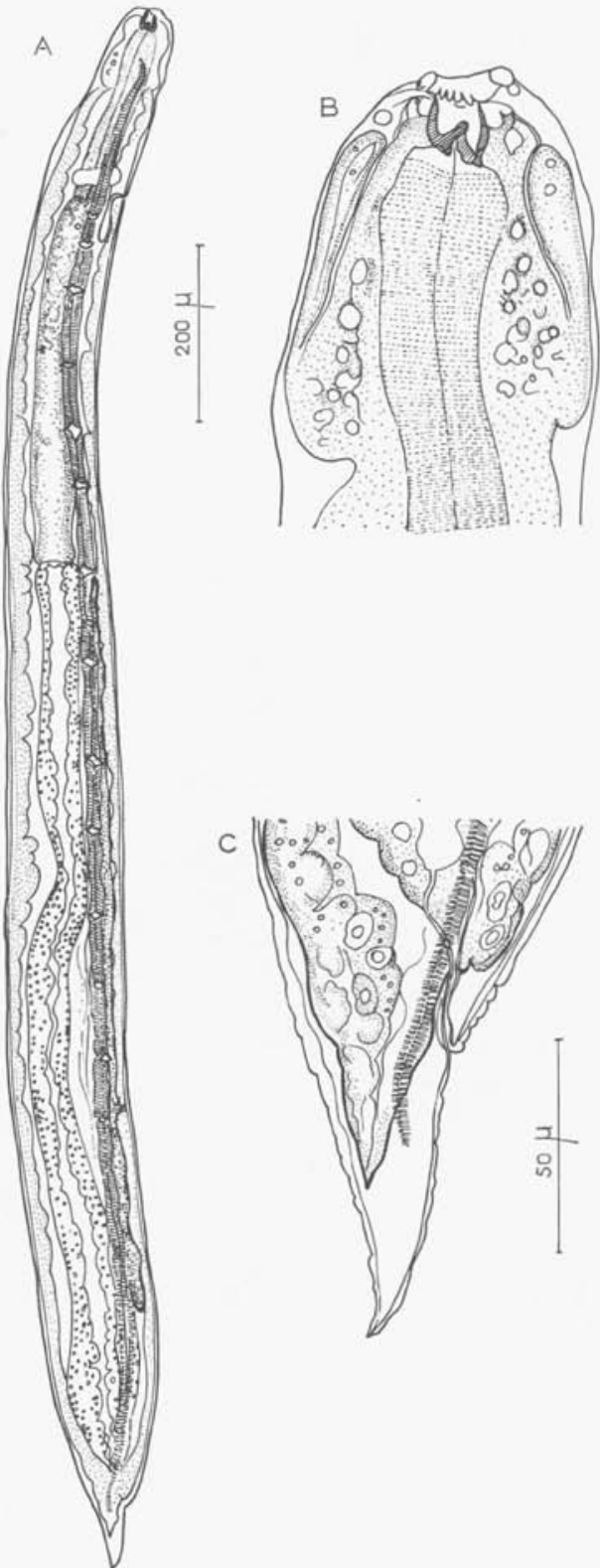

Fig. 5 - A: larve femelle, fin du $4^{e}$ stade, vue latérale. B : décollement de l'exuvie dans la région céphalique. $\mathrm{C}$ : idem, région caudale. A : éch. $200 \mu$. B, C: éch. $50 \mu$ 

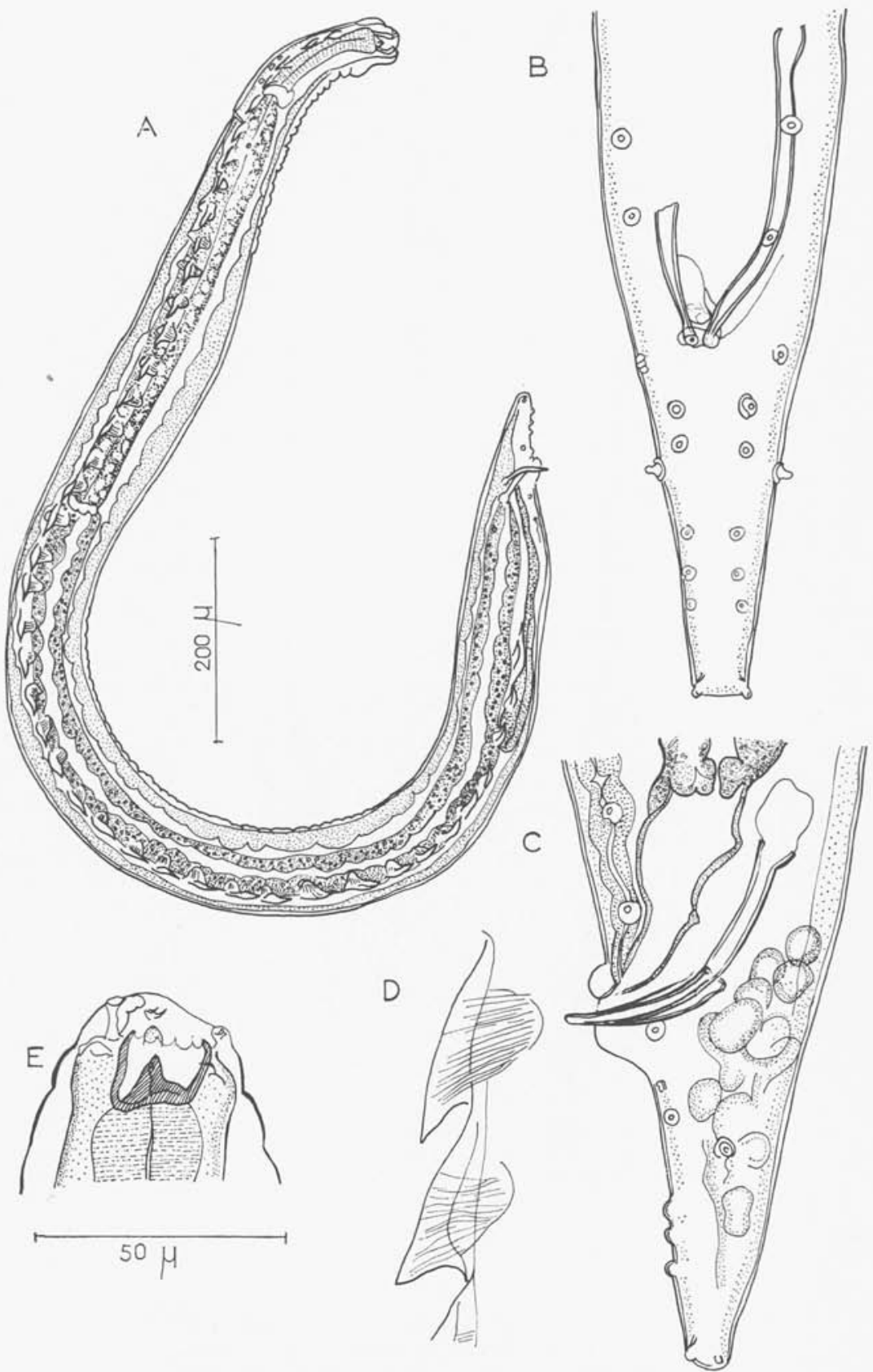

Fig. 6 - A : jeune mâle, $108 \mathrm{~h}$ après l'infestation chez l'hôte définitif. B, C : détail des vues ventrales et latérales de la bourse caudale. D: détail des peignes cuticulaires, vue ventrale. E : extrémité antérieure, vue latérale gauche. A : éch. $200 \mu$. B, C, D, E : éch. $50 \mu$ 
- Pterygodermatites coloradensis (Hall, 1916) est essentiellement parasite de Rongeurs Cricetidae d'Amérique du Nord. Son cycle biologique est réalisé par Oswald 1958 par l'infestation de Dictyoptères, d'Orthoptères et de Coléoptères.

- P. zygodontomis (Quentin, 1967), parasite d'un Cricetidae du Brésil, évolue expérimentalement chez un Dermaptère (Quentin 1969).

Le cycle biologique de $P$. desportesi (Chabaud et Rousselot, 1956) est comparable à ceux des espèces précédentes : trois stades larvaires sont observés chez l'hôte intermédiaire et il existe, selon nous, un quatrième stade chez l'hôte définitif. (Nous avons vu, dans le cycle de $P$. zygodontomis, une mue extrêmement précoce, séparant le troisième du quatrième stade, qui survient chez le Rongeur dans les deux premiers jours du développement. Nous pensons que le phénomène existe chez toutes les espèces mais passe facilement inaperçu).

Ce quatrième stade donne naissance à l'adulte cinq jours environ après l'infestation.

Après quatre jours et demi de développement chez Praomys morio, nous avons récolté à la fois une larve femelle du quatrième stade et un jeune mâle.

L'évolution chez l'hôte intermédiaire est rapide. A une température voisine de $22-26^{\circ} \mathrm{C}$ pour $P$. coloradensis, de $25^{\circ} \mathrm{C}$ pour $P$. zygodontomis, le troisième stade larvaire est obtenu après 13 jours de développement chez l'Insecte. A $22^{\circ} \mathrm{C}$, la seconde mue survient chez $P$. desportesi entre le $17^{\circ}$ et le $24^{\circ}$ jour.

Les larves du troisième stade de Rictulariidae sont remarquablement petites comme en témoigne le tableau ci-dessous où sont indiquées les longueurs et les largeurs maximum en microns des trois premiers stades. Elles se différencient donc, par la taille, des autres larves infestantes de Spirurides connues jusqu'à présent, qui mesurent plus de $1 \mathrm{~mm}$.

\begin{tabular}{|c|c|c|c|}
\hline & $1^{\text {er }}$ STADE & $2^{\circ}$ STADE & $3^{e}$ STADE \\
\hline$R$, cristata $\ldots \ldots \ldots \ldots \ldots \ldots$ & $451 \times 22$ & $672 \times 64$ & $896 \times 51$ \\
\hline P. coloradensis ............. & $326 \times 15,6$ & $489 \times 36$ & $593 \times 61$ \\
\hline P. zygodontomis $\ldots \ldots \ldots \ldots$ & $315 \times 36$ & $640 \times 70$ & $710 \times 70$ \\
\hline P. desportesi $\ldots \ldots \ldots \ldots \ldots \ldots$ & $380 \times 20$ & $550 \times 37$ & $725 \times 65$ \\
\hline
\end{tabular}

La structure céphalique des larves du troisième stade de $P$. desportesi présente un cas d'orthogénèse. La tête de la larve (fig. $4 \mathrm{~A}$ ) ne ressemble pas à celle de l'adulte correspondant mais à celle des adultes des espèces néarctiques les plus primitives. Ces caractères céphaliques larvaires: capsule buccale terminale, papilles labiales internes réunies entre elles par des cordons d'ornementations cuticulaires, trois dents œsophagiennes d'égale importance et denticulées, sont, en effet, ceux des adultes des espèces nord-américaines du groupe coloradensis. 
Cette récapitulation ontogénétique est un phénomène déjà connu chez les Nématodes Ascarides et Acuarides. Elle paraît ici confirmer l'évolution phylogénétique des Rictulaires que nous avons tenté de tracer dans un travail précédent.

\section{Bibliographie}

Chabaud (A.-G.) et Rousselot (R.), 1956. - Deux nouveaux Rictularia (Nematoda, Thelaziidae) d'Afrique équatoriale. Ann. Parasit. Hum. Comp., 31 (3), p. 255-265, fig. 1 A $5 \mathrm{~F}$.

Morosov (J.-F.), 1960. - Sur la biologie du cycle de Rictularia amurensis Schulz, 1927 (Nematoda Rictulariidae) (En russe). Inst. Ped. Gork., 27, s. Helm., (2), p. 17-28, fig. 1-9.

Oswald (V. H.), 1958. - Studies on Rictularia coloradensis Hall, 1916 (Nematoda: Thelaziidae). I. Larval development in the intermediate host. Trans. Amer. Micr. Soc., 77 (3), p. 229-240, fig. 1-13.

-, 1958. - Studies on Rictularia coloradensis Hall, 1916 (Nematoda: Thelaziidae). II. Development in the definitive host. Ibid., 77 (4), p. 413-422, fig. 1-4, graph. 1-2, tabl. 1-3.

Quentin (J.-C.), 1965. - Spirurides de Rongeurs de la Station Expérimentale de La Maboké. Bull. Mus. Nat. Hist. Nat., 37 (4), p. 690-700, fig. 1-3.

-, 1969. - Essai de classification des Nématodes Rictulaires. Mém. Mus. Nat. Hist. Nat., nelle sér., sér. A. Zool., 54 (2), p. 55-115, fig. 1-27. 